\section{$\underset{\substack{\text { hommes } \\ \text { \& migrations }}}{ }$}

\section{Hommes \& migrations}

Revue française de référence sur les dynamiques

migratoires

$1288 \mid 2010$

Langues et migrations

\title{
Du bilinguisme familial chez les enfants
}

\section{Francine Couëtoux-Jungman et Claire Extramiana}

\section{(2) OpenEdition \\ Journals}

\section{Édition électronique}

URL : http://journals.openedition.org/hommesmigrations/866

DOI : 10.4000/hommesmigrations.866

ISSN : 2262-3353

\section{Éditeur}

Musée national de l'histoire de l'immigration

\section{Édition imprimée}

Date de publication : 1 novembre 2010

Pagination : 80-85

ISSN : 1142-852X

\section{Référence électronique}

Francine Couëtoux-Jungman et Claire Extramiana, "Du bilinguisme familial chez les enfants », Hommes \& migrations [En ligne], 1288 | 2010, mis en ligne le 29 mai 2013, consulté le 19 avril 2019. URL : http://journals.openedition.org/hommesmigrations/866 ; DOI : 10.4000/ hommesmigrations.866 


\section{Du bilinguisme familial chez les enfants}

Entretien avec Francine Couëtoux-Jungman, psychologue clinicienne réalisé par Claire Extramiana, délégation générale à la langue française et aux langues de France

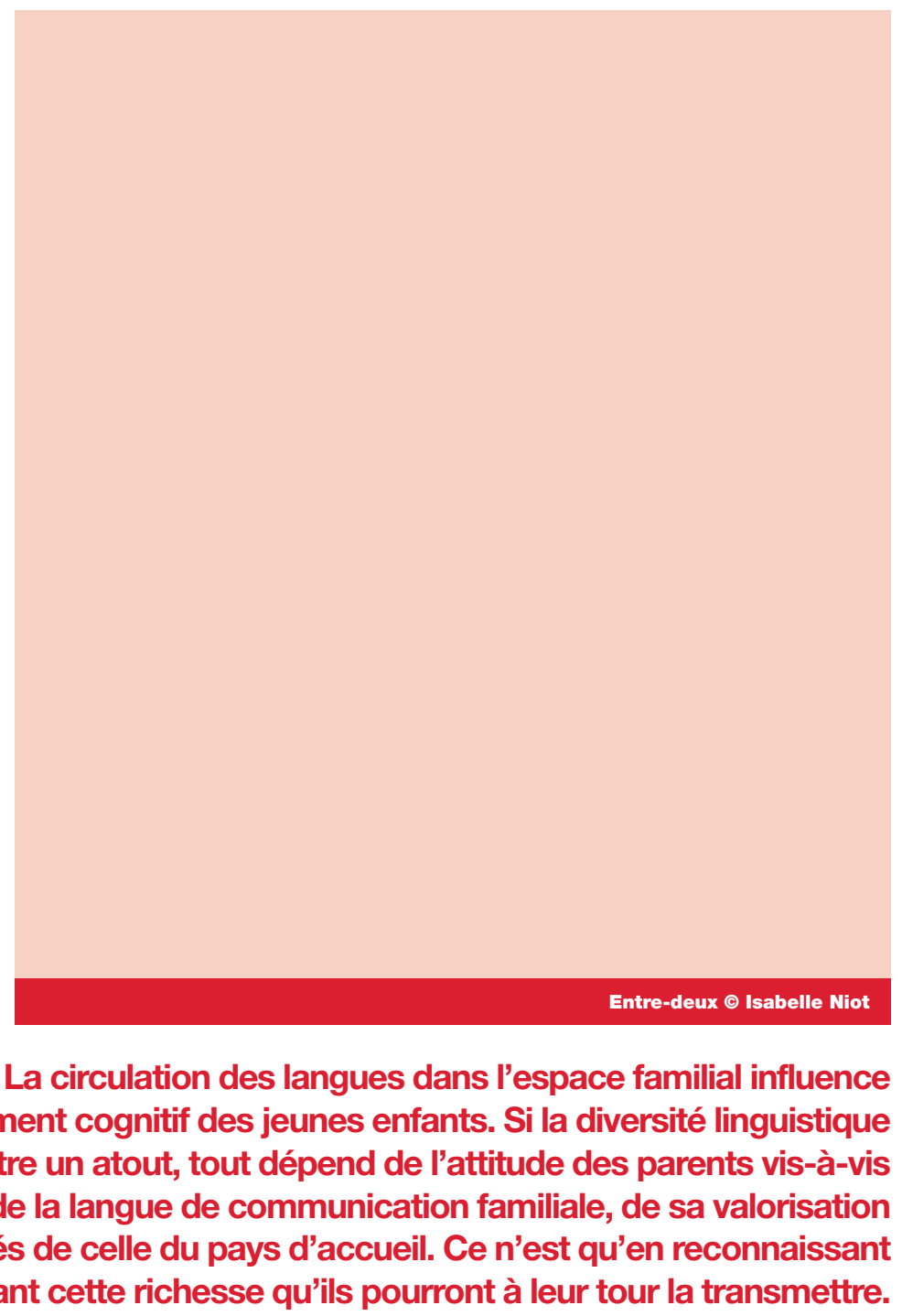




\section{En tant que psychologue clinicienne, vous êtes spécialiste du bilin- guisme dans les interactions précoces, c'est-à-dire la communication parents-enfant. Depuis quand les psychologues cliniciens s'intéressent- ils à cette question ? Comment en êtes-vous venue à vous y intéresser vous-même?}

Francine Couëtoux-Jungman : En fait, je suis avant tout spécialiste de la psychopathologie du développement du jeune enfant et de la psychothérapie mère-bébé. C'est au cours de mon travail clinique que j'ai été confrontée à la question des interactions précoces des mères avec leur bébé dans des contextes de bilinguisme ou de plurilinguisme familial. Chez les spécialistes de l'enfance travaillant avec les enfants migrants, il y a les courants de l'ethnopsychiatrie et de l'ethnopsychanalyse qui s'intéressent aux langues. Je citerai le travail de Marie-Rose Moro au sein du service de psychopathologie de l'enfant et de l'adolescent de l'hôpital Avicenne, à Bobigny, et je renvoie à ses manuels ainsi qu'au numéro "L'enfant et les langues" de la revue L'Autre, qu'elle dirige. D'autres praticiens mènent actuellement des travaux de recherche sur cette question.

Je me focaliserai ici sur le facteur linguistique, étant bien entendu qu'il ne peut être question, lorsque nous recevons les enfants et leurs parents, de nous limiter à la question du contexte linguistique dans lequel évoluent les enfants.

C'est dans le cadre d'un travail de prévention et de soins pour des enfants scolarisés de 3 à 6 ans, qui présentaient des retards de langage et des troubles psychomoteurs, que je me suis intéressée au départ au langage des enfants issus d'un environnement bilingue ou plurilingue. L'écoute de leurs parents m'a permis de me pencher sur le bilinguisme, qui est une question complexe. C'est ensuite que j'ai participé à la création de l'unité petite enfance et parentalité Vivaldi à Paris (XII ${ }^{\mathrm{e}}$ arrondissement), rattachée au service de psychiatrie de l'enfant et de l'adolescent de la Pitié-Salpêtrière où j'exerce actuellement. Cette unité est ouverte aux futurs parents et aux parents avec leur bébé, de la naissance à 3 ans. Nous y accueillons plus d'un quart d'enfants de parents ayant une langue première autre que le français. Les enfants de contexte bilingue sont reçus à l'accueil thérapeutique en général plus tard que les enfants de contexte monolingue (que nous voyons la première fois vers 1 an), c'est-à-dire vers 2 ans.

J'observe dans ma pratique que l'accès entravé à la langue des parents retarde l'acquisition du langage chez l'enfant; le fait que les parents ne communiquent pas avec leur enfant dans leur langue, alors qu'ils la parlent entre eux, et sont peu à l'aise dans la langue française, peut produire des inhibitions verbales chez lui qui,à leur tour, ont des répercussions négatives sur la construction du langage chez l'enfant.S'intéresser aux langues est donc essentiel. 


\section{Pouvez-vous nous présenter des exemples d'inhibitions verbales ?}

F. C.-J. : Il y a le cas d'un petit garçon de 3 ans et demi. Sa mère, bilingue, disait ne pas le comprendre quand il parlait. Elle s'adressait toujours à lui en français alors qu'elle s'exprimait davantage dans sa première langue avec son mari, sa propre mère et ses amis. Le travail en entretien a porté sur l'écoute de l'enfant par la mère. En effet, l'enfant disait des mots dans la langue première maternelle, ce que n'attendait ni n'entendait sa mère puisqu'elle ne lui parlait que français. Cette dernière a alors pu reconnaître ces mots prononcés par son enfant et reformuler ce qu'elle avait compris, cela s'est avéré stimulant pour l'apprentissage du langage chez l'enfant.

Une autre mère me disait s'adresser, de la même manière, uniquement en français à son enfant de 4 ans afin qu'il parle français et s'intègre plus facilement à l'école. Or cet enfant avait un retard de langage et faisait d'impressionnantes colères à la maison, ce qui avait motivé une consultation dans notre centre. Cette mère parlait principalement sa langue d'origine dans ses relations avec le reste de sa famille, partageant la même langue première avec son mari comme dans la situation précédente. Ce n'est que dans des moments de débordement que cette première langue venait à son insu s'imposer dans sa relation à son enfant. L'amener à s'adresser dans cette langue première à son enfant dans des contextes plus positifs a eu un effet très bénéfique sur leur relation.

\section{En quoi le refoulement était-il négatif dans le cas présent ?}

F. C.-J.: Quand la langue refoulée resurgit dans les moments de difficultés relationnelles, l'enfant associe de ce fait cette langue à des émotions négatives. Nous conseillons aux parents, lors des entretiens, de parler aussi la langue d'origine dans des moments où ils sont bien, comme je viens de l'expliquer.

\section{Le bilinguisme constitue-t-il d'après vous un frein ou un atout pour le développement du petit enfant ?}

F. C.-J. : Le bilinguisme est plutôt un atout pour l'enfant. Il est aujourd'hui reconnu comme favorable au développement des capacités attentionnelles, des compétences métalinguistiques, des facultés d'abstraction. En revanche, si l'enfant est intégré aussi bien à la langue familiale qu'à celle du pays d'accueil, il peut grandir dans un métissage linguistique et culturel très favorable. Par contre, si la langue familiale n'est pas reconnue et reste circonscrite à l'univers familial de façon très fermée, il y a un risque de clivage pour l'enfant. On l'observe dans des difficultés comme le mutisme extra-familial de certains jeunes enfants migrants : ces enfants parlent en famille, mais sont mutiques à l'extérieur. Bien sûr, il faut distinguer les parents à l'aise dans leur bilinguisme et ceux qui le sont moins. Ou qui sont mal à l'aise dans la langue du pays d'accueil, surtout s'ils choisissent de la parler comme unique langue à leur enfant. Linhibition langagière 
des parents risquant alors de produire des retards de langage chez l'enfant. D'une manière générale on peut dire que les avis des professionnels de la petite enfance et de l'enfance (l'école) sont encore divergents et qu'il reste difficile de conseiller les familles dans ce domaine. Mais ce qui compte, c'est que les parents aient des informations sur le bilinguisme et qu'ils puissent élaborer leurs choix linguistiques de façon éclairée.

\section{Pouvez-vous nous expliquer ce qui se passe dans les familles bilingues - ou plurilingues - pour l'enfant en âge d'apprendre à parler ?}

F. C.-J. : Pour les mères qui maintiennent leur langue dans la communication avec leur bébé, ce contexte n'engendre pas de difficulté particulière pour l'enfant en termes d'acquisition du langage. L'enfant va parler la langue de la mère avec laquelle l'interaction verbale ne pose pas de problème. Être à l'aise dans sa langue est une condition de l'interaction réussie avec le jeune enfant.

Notre unité a mis en place un projet pour permettre la pratique des langues parentales au cours d'activités de socialisation des parents avec leur bébé. Des associations proposent des rencontres pour les parents et leurs enfants dans des langues étrangères, comme le "café bilingue" par exemple, dont je fais partie au titre du conseil scientifique. On rencontre des difficultés dans des contextes où les parents parlent entre eux une langue qu'ils n'emploient pas avec leur enfant. Pour les enfants nés en France dont les parents sont en train d'acquérir la langue française, on peut parler de risque d'inhibition verbale ou langagière si la langue première est délaissée au profit du français, surtout pour les enfants qui ne bénéficient pas d'une exposition supplémentaire au français à travers notamment le mode de garde (halte-garderie, crèche).

Quand on maîtrise moins bien une langue seconde, on inhibe la parole du fait de cette difficulté de langage. Ne pas mettre de mots oblige au langage gestuel qui génère des relations très compliquées dès qu'on dépasse les relations proximales, c'est-à-dire en général dès que l'enfant marche. Le langage corporel tend à compenser ce déficit du langage, or il est très insuffisant du point de vue de la communication. Il ne permet pas, quand les messages se complexifient, d'expliquer, de prévenir, d'argumenter et peut déboucher sur un rapport de force entre l'adulte qui demande et l'enfant qui résiste. Dans cette situation, l'enfant va avoir des réactions où le corps exprimera l'agitation, l'opposition, la colère. Ce comportement vient s'ajouter à un déficit de compréhension du langage, d'écoute et d'attention.

\section{Comment prenez-vous en compte l'environnement linguistique de vos jeunes patients dans votre travail clinique ?}

F. C.-J. : J'attire l'attention des parents sur la question des langues, les choix conscients et moins conscients. Les parents bilingues nous disent parfois ne parler 
que le français à leur enfant afin de l'aider à mieux s'intégrer. Ils peuvent comprendre que la langue qu'ils parlent entre eux, ou qu'ils parlent au téléphone avec les parents ou avec des amis, concerne directement l'enfant. En effet, celui-ci entend ces échanges très importants et riches pour ses parents. Si cette langue lui est aussi adressée, il sera intégré dans la communication familiale. Dans le cas contraire, il risque d'être exclu de cette communication intrafamiliale et de se désintéresser du langage. Mettre en place avec eux un diagnostic de leurs pratiques linguistiques est très important pour la résolution du conflit des langues en présence, car le bilinguisme harmonieux existe. Les inhibitions langagières ne concernent pas que les mères bilingues, bien évidemment, mais il y a une aide spécifique à mettre en place pour elles.

Je travaille avec des imagiers ou des livres pour enfants que j'ai acquis dans différentes langues. On peut se les procurer à Paris dans les librairies étrangères ou lors de voyages, ou bien aussi acquérir des imagiers multilingues. Le support des atlas est aussi très favorable aux échanges avec les mères et leur enfant sur leur histoire, leur trajectoire, leur culture, leur langue, ou les langues qu'elles ont parlées et apprises. Il m'arrive souvent d'inciter les mamans à parler à leur enfant ou à chanter une chanson dans leur langue première, celle de leur propre petite enfance. Cela, afin de retrouver des émotions et de communiquer différemment avec leur enfant. Mon intérêt pour leur langue la valorise et permet de la faire exister dans l'espace social et pas seulement dans l'espace familial. Cela permet à l'enfant de reconnaitre son contexte de bilinguisme et d'y être plus à l'aise.

\section{Votre thèse de doctorat prolonge votre travail de clinicienne. Comment fait-elle avancer la connaissance sur ce sujet ?}

F. C.-J. : Cette thèse, actuellement en cours, traite de l'influence de contextes de bilinguisme et de plurilinguisme sur les interactions précoces des parents avec leur bébé et le développement du jeune enfant. Ce travail comprend: une étude quantitative d'une population de parents et d'enfants fréquentant notre accueil thérapeutique, avec une comparaison des populations monolingues et bilingues puis bilingues avec maintien total ou partiel ou non-maintien de la langue maternelle ; une étude qualitative des relations précoces des parents avec leur enfant en contexte de bilinguisme. Une des hypothèses est que le maintien de la langue maternelle, même partiel, favorise l'accès à la communication précoce et au langage. Une autre hypothèse porte sur le type de bilinguisme : il semble que les parents ayant la même langue première ont tendance à moins la maintenir dans leurs échanges avec l'enfant que si l'un des deux est francophone. Sans doute du fait de leur crainte que leur enfant connaisse des difficultés d'intégration à l'école maternelle si aucun des deux parents ne lui parle français. 


\section{II y a des bilinguismes (ou plurilinguismes) valorisés et prestigieux, et d'autres qui le sont moins, quand une des langues est jugée non pres- tigieuse. Cela vaut-il pour certaines familles que vous rencontrez?}

F. C.-J. : Le prestige de la langue joue un rôle, indéniablement, de même que le regard que les parents portent sur leur langue, ce qui renvoie aux langues minorées. Leur non-prise en compte produit des effets négatifs en termes d'interactions verbales. Lors de nos accueils thérapeutiques, nous avons reçu une mère de langue première étrangère en difficulté avec son enfant. Le contexte de bilinguisme paraissait a priori simple car le père parlait français, mais il s'est avéré plus complexe. En effet, l'enfant était souvent en contact avec la langue locale de ses grands-parents maternels, puisqu'il séjournait régulièrement chez eux. Il entendait aussi très souvent sa mère parler au téléphone cette langue locale avec ses amis, sa famille. Or sa mère ne lui parlait que la langue nationale qui se trouvait être une langue plus valorisée internationalement.

Tout cela a un rapport avec l'estime de sa langue, souvent liée à l'estime de soi. On observe que certaines mères n'osent pas parler leur langue qu'elles estiment n'être qu'un patois ou un dialecte. Il s'avère que si elles ne maîtrisent pas suffisamment la langue nationale de leur pays, elles vont finalement très peu parler à leur enfant. Cette situation se produit dans le cas des langues locales minoritaires. Mais, je le répète, la reconnaissance de la langue parentale, sa pratique avec l'enfant dès la naissance, dans les premiers liens puis au-delà, garantissent une relation plus saine, non exclusive de l'acquisition du français, dès lors que le langage est acquis.

\section{Bibliographie}

- Abdelilah-Bauer Barbara, Le défi des enfants bilingues, grandir et vivre en parlant plusieurs langues, Paris, La Découverte, 2006.

- Bijeljac-Babic R, "Acquisition de la phonologie et bilinguisme précoce", in Kail Michel, Fayol Michel, L'Acquisition du langage, Paris, PUF, 2000, pp. 169-192.

- Couëtoux F, "Interactions précoces et bilinguisme", in Le Journal des psychologues, n 256, avril 2008.

- Couëtoux F, "Le temps de l'accueil", Le Journal des psychologues, n 211, octobre 2003.

- Couëtoux F, "Auprès des parents et de leur bébé en difficulté", in Le Journal des psychologues n 175 , mars 2000.

- De Houwer Annick, "Le développement harmonieux ou non harmonieux du bilinguisme de l'enfant au sein de la famille", in Langage et Société, vol. 2, n 116, 2006, pp. 29-49.

- Deprez Christine, "La transmission des langues d'immigration dans l'enquête sur l'histoire familiale annexée au recensement de 1999", in Migrations et plurilinguisme en France, Cahiers de l'Observatoire des pratiques linguistiques, Délégation générale à la langue française et aux langues de France, $n^{\circ}$ 2, 2008, pp. 34-42.

- Hagège Claude, L'Enfant aux deux langues, Paris, Odile Jacob, 2005.

- Hamers J. F., Blanc J., Bilingualité et bilinguisme, Bruxelles, Pierre Mardaga, 2008.

- Héran François, "Une approche quantitative de l'intégration linguistique en France", Hommes et Migrations, $\mathrm{n}^{\circ}$ 1252, "Langues de France", 2004, pp. 10-24.

- Moro Marie-Rose, De La Noe Q., Mouchenik Y. (éd.), Manuel de psychiatrie transculturelle, Paris, La Pensée sauvage, 2004.

- Moro Marie-Rose, Riand R., Plard V. (éd.), Manuel de psychopathologie du bébé et de sa famille, Paris, La Pensée sauvage, 2010.

- Siguan M., Mackey W.F, Éducation et bilinguisme, Unesco, Lausanne, Delachaux et Niestlé, 1986.

- Rabain D., Aidane E., Couëtoux F., Marie P. et Mazet P., "Le travail avec des bébés et leurs parents au sein d'une unité spécialisée", in Angel P., Mazet P., Guérir les souffrances familiales, Paris, PUF, 2004. 